\title{
Thermal expansion of La-based BMG studied by in situ high-energy $\mathrm{X}$-ray diffraction
}

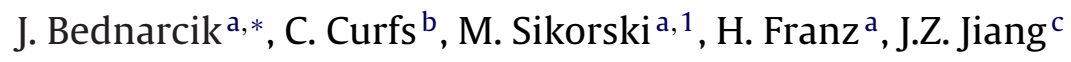 \\ a Deutsches Elektronen-Synchrotron DESY, Notkestraße 85, D-22603 Hamburg, Germany \\ ${ }^{\mathrm{b}}$ European Synchrotron Radiation Facility ESRF, BP 220, 38043 Grenoble, France \\ ${ }^{c}$ International Center for New-Structured Materials ICNSM, Department of Materials Science and Engineering, Zhejiang University and \\ Laboratory of New-Structured Materials, Zhejiang University, Hangzhou 310027, PR China
}

\section{A R T I C L E I N F O}

\section{Article history:}

Received 3 July 2009

Received in revised form 7 January 2010

Accepted 10 February 2010

Available online 20 February 2010

\section{Keywords:}

Bulk metallic glasses

Glass transition

X-ray diffraction

Thermal expansion

\begin{abstract}
A B S T R A C T
The present work focuses on the study of the thermal stability of $\mathrm{La}_{62} \mathrm{Al}_{14}\left(\mathrm{Cu}_{5 / 6} \mathrm{Ag}_{1 / 6}\right)_{14} \mathrm{Ni}_{5} \mathrm{Co}_{5}$ (at.\%) bulk metallic glass (BMG) using the state-of-the-art high temperature in situ hard X-ray scattering techniques available at the undulator beamline ID11 of ESRF (Grenoble, France). Constant-rate heating experiments using a fast X-ray camera (time resolution of $3 \mathrm{~s}$ ) reveal detailed information about the thermal stability of the La-based BMG. Analyzing diffraction patterns in a reciprocal space yields thermal expansion of amorphous alloy providing insight about the thermally activated relaxation effects and glass transition. We found that in the region $20 \mathrm{~K}$ below the glass transition $\left(T_{g}=420 \mathrm{~K}\right)$ thermal expansion curve exhibits negative slope which indicates annealing-out of the quenched-in free volumes. The glass transition appears as a brake in the value of coefficient of the volume thermal expansion $\alpha_{\mathrm{th}}$. Furthermore, real space analysis based on reduced pair distribution function $G(r)$ allows to trace the changes in the local atomic structure of amorphous material during constant-rate heating.
\end{abstract}

(c) 2010 Elsevier B.V. All rights reserved.

\section{Introduction}

Bulk metallic glasses represent relatively new class of materials exhibiting unique mechanical, chemical, electronic and magnetic properties which strongly differ from the ones of their crystalline counterparts [1]. These multi-component metallic alloys combine high glass-forming ability with low cooling rates below $100 \mathrm{~K} / \mathrm{min}$ which allow formation of few mm thick samples by conventional mould casting $[2,3]$. Structurally, bulk metallic glasses can be classified as disordered materials. X-ray diffraction (XRD) using high-energy photons has proven to be well suited for describing the structure of disordered systems such as BMGs. Time-resolved in situ X-ray diffraction experiments may nowadays be performed at high-brilliance synchrotron radiation sources for a variety of conditions which help to elucidate the structure-property relations.

Aim of this work is to study the thermal stability of $\mathrm{La}_{62} \mathrm{Al}_{14}\left(\mathrm{Cu}_{5 / 6} \mathrm{Ag}_{1 / 6}\right)_{14} \mathrm{Ni}_{5} \mathrm{Co}_{5}$ BMG in the temperature range $300-800 \mathrm{~K}$ using in situ high-energy X-ray diffraction. The special emphasis is placed at the study of its thermal expansion. Both,

\footnotetext{
* Corresponding author.

E-mail address: jozef.bednarcik@desy.de (J. Bednarcik).

1 Present address: Advanced Photon Source, Argonne National Laboratory, Argonne, IL 60439, USA.
}

reciprocal space as well as real space analysis are employed in order to resolve the structural changes occurring in the vicinity of the glass transition.

\section{Experimental procedures}

Amorphous ingots with nominal composition of $\mathrm{La}_{62} \mathrm{Al}_{14}\left(\mathrm{Cu}_{5 / 6} \mathrm{Ag}_{1 / 6}\right)_{14} \mathrm{Ni}_{5} \mathrm{Co}_{5}$ (at.\%) were prepared by suction-casting into copper mold (with diameter of $0.9 \mathrm{~mm}$ ) under a purified argon atmosphere. The purities of the elements ranged from 99.5 to 99.98 at.\%. A differential scanning calorimeter (NETZSCH DSC 404C) was used to detect the crystallization and melting behavior of as-prepared BMG under a continuous argon flow at a heating rate of $20 \mathrm{~K} / \mathrm{min}$. Temperature evolution of the as-casted rods was continuously followed using a high-energy monochromatic beam on the ID11 undulator beamline at the ESRF (Grenoble, France). The rod sample having diameter of $0.9 \mathrm{~mm}$ and length of $4 \mathrm{~mm}$ was sealed into quartz capillary. Annealing was done in Ar-protective atmosphere using a computer-controlled Linkam THMS 600 hot stage. Heating and cooling rates were $20 \mathrm{~K} / \mathrm{min}$. The synchrotron radiation wavelength was set to $\lambda=0.01409 \mathrm{~nm}$ (photon energy $88 \mathrm{keV}$ ). The sample was illuminated with well collimated incident beam having a cross-section of $0.7 \mathrm{~mm} \times 0.3 \mathrm{~mm}$. The diffracted photons were collected up to a maximum wave vector momentum transfer $q=120 \mathrm{~nm}^{-1}(q=4 \pi \sin \theta / \lambda)$ using a fast two-dimensional CCD detector (FRELON 2k16, $2048 \times 2048$ pixels, each pixel having effective size of $50 \mu \mathrm{m} \times 50 \mu \mathrm{m}$ ) carefully aligned orthogonal to the X-ray beam. The delay between consecutive patterns, due to read-out time of the CCD and write-time to hard-disc was $3 \mathrm{~s}$. Two-dimensional diffraction patterns were integrated using the FIT2D software package [4]. The observed X-ray scattering intensities $I^{\text {obs }}(q)$ were corrected for background, absorption, polarization, incoherent and multiple scattering effects (all procedures are described in reference [5]). Using the Faber-Ziman formalism [6] the total structure factor $S(q)$ is obtained from the coherently scattered intensity 


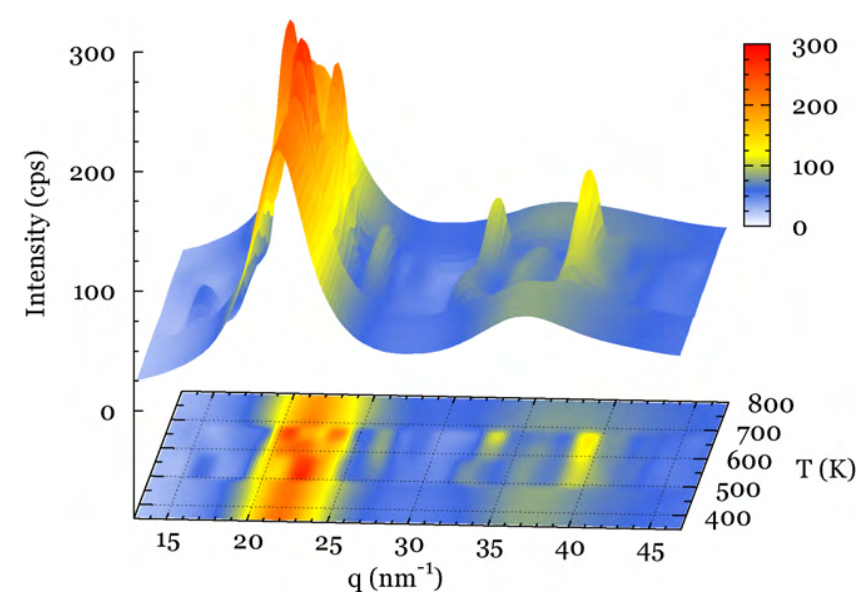

Fig. 1. The series of $\mathrm{X}$-ray diffraction patterns obtained in transmission mode on $\mathrm{La}_{62} \mathrm{Al}_{14}\left(\mathrm{Cu}_{5 / 6} \mathrm{Ag}_{1 / 6}\right)_{14} \mathrm{Ni}_{5} \mathrm{Co}_{5}$ BMG during constant-rate heating $(20 \mathrm{~K} / \mathrm{min})$ up to $800 \mathrm{~K}$.

$I^{\operatorname{coh}}(q)$

$S(q)=1+\frac{I^{\operatorname{coh}}(q)-\left\langle f^{2}\right\rangle}{\langle f\rangle^{2}}$

where $\langle f\rangle=\sum_{i=1}^{n} c_{i} f_{i}(q)$ and $\left\langle f^{2}\right\rangle=\sum_{i=1}^{n} c_{i} f_{i}^{2}(q)$, in which $c_{i}$ corresponds to the atomic fraction of the component $i$ having X-ray atomic scattering factor $f_{i}(q)$. The reduced pair distribution function, $G(r)$, can be obtained through a sine Fourier transformation

$G(r)=\frac{2}{\pi} \int_{0}^{\infty} q[S(q)-1] \sin (q r) \mathrm{d} q$.

\section{Results and discussion}

Fig. 1 shows series of X-ray diffraction patterns of $\mathrm{La}_{62} \mathrm{Al}_{14}\left(\mathrm{Cu}_{5 / 6} \mathrm{Ag}_{1 / 6}\right)_{14} \mathrm{Ni}_{5} \mathrm{Co}_{5}$ rod acquired during constant-rate heating $(20 \mathrm{~K} / \mathrm{min})$ up to $800 \mathrm{~K}$. The room temperature patterns exhibit broad diffuse maximum at $Q \approx 21.95 \mathrm{~nm}^{-1}$ together with relatively weak oscillations, which gradually disappear with increasing $q$ up to $120 \mathrm{~nm}^{-1}$. The first look on series of diffraction data presented in Fig. 1 suggests it consists of three distinct temperature regions, each characterizing different state of a material: (i) amorphous solid, (ii) crystalline state and finally (iii) amorphous liquid. The same structural transformation sequence is confirmed by DSC. As can be seen from DSC curve presented in Fig. 2a, investigated alloy reveals the presence of relatively wide supercooled liquid region $\Delta T_{x}=T_{x}-T_{g}=60 \mathrm{~K}$, which starts with the glass temperature $T_{g}=422 \mathrm{~K}$ and finishes with the crystallization temperature $T_{x}=482 \mathrm{~K}$. Melting $T_{m}$ and liquidus $T_{L}$ temperatures determined by DSC are $642 \mathrm{~K}$ and $727 \mathrm{~K}$, respectively (see Fig. 2b).

As proposed by Yavari et al. [7] the third power of the principal diffuse peak position $Q$ scales with the coefficient of the volumetric thermal expansion $\alpha_{\text {th }}$ of amorphous material as in

$$
\left\{\frac{Q\left(T_{0}\right)}{Q(T)}\right\}^{3}=\left\{\frac{V(T)}{V\left(T_{0}\right)}\right\}=1+\alpha_{\mathrm{th}}\left(T-T_{0}\right)
$$

where $V(T) / V\left(T_{0}\right)$ is the reduced mean atomic volume at $T$, with the reference temperature $T_{0}$ (in this work $T_{0}=320 \mathrm{~K}$ ). Furthermore it was shown that the atomic volume change computed using Eq. (3) is equivalent to the macroscopic volume change obtained by dilatometry [7]. It should be noted here that for isotropic materials, the length thermal expansion coefficient $\beta_{\text {th }}$ is one third the volumetric coefficient $\alpha_{\mathrm{th}}$, i.e. $\alpha_{\mathrm{th}}=3 \beta_{\mathrm{th}}$. Since observed changes in the principal peak position $Q$ with the temperature are relatively small
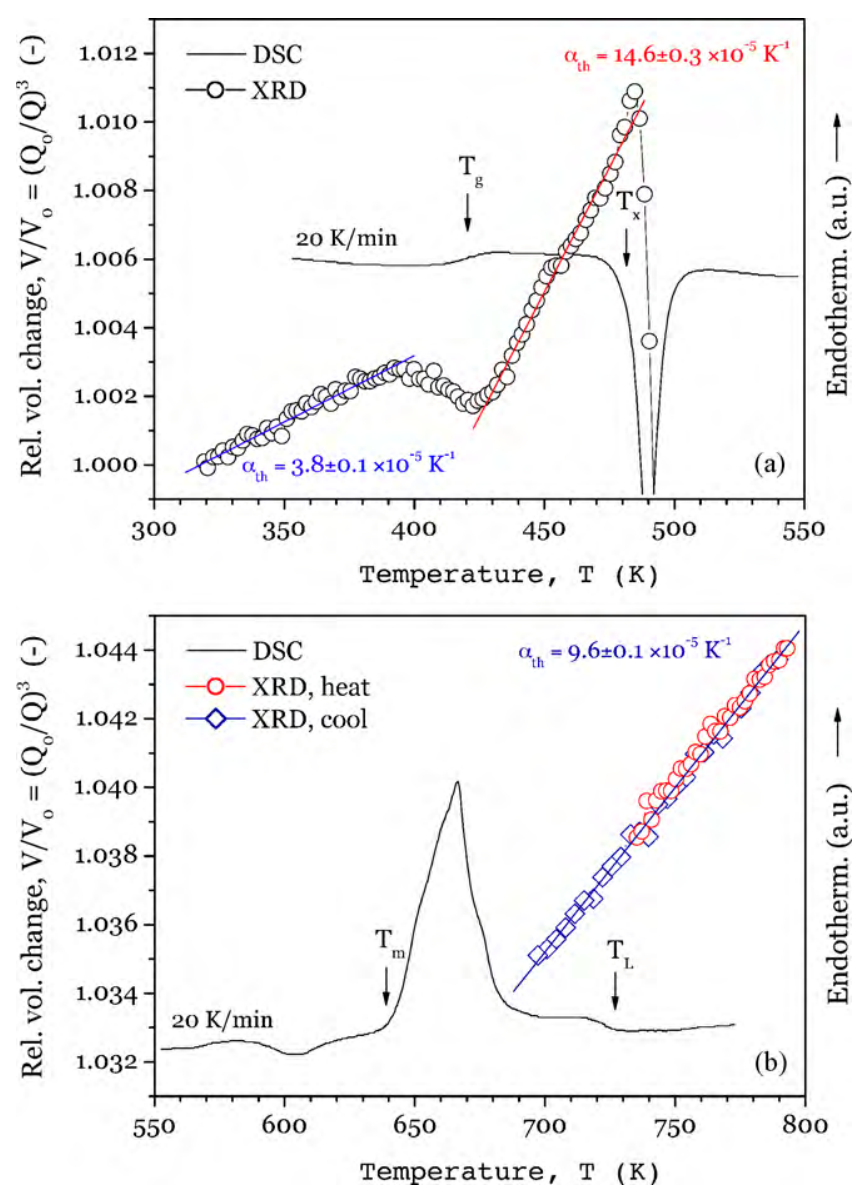

Fig. 2. DSC curve and thermal expansion curves for $\mathrm{La}_{62} \mathrm{Al}_{14}\left(\mathrm{Cu}_{5 / 6} \mathrm{Ag}_{1 / 6}\right)_{14} \mathrm{Ni}_{5} \mathrm{Co}_{5}$ BMG (a) below crystallization and (b) above melting temperature.

and thus quite sensitive to the noise in data, one should include in the peak position (center of mass) determination procedure as many points so that the influence of an individual datapoint on the resulting peak position is minimized. In the present study the principal diffuse peak ( $q$-range between 15 and $30 \mathrm{~nm}^{-1}$ ) was modelled using the pseudo-Voigt function and linear background. Tracing the peak position of the principal diffuse peak below crystallization temperature $T_{x}$ we obtained the thermal expansion of La-based BMG. As can be seen from Fig. 2a an early stages of heating can be characterized as the linear volume expansion with the coefficient of volume thermal expansion $\alpha_{\mathrm{th}}=3.8 \pm 0.1 \times 10^{-5} \mathrm{~K}^{-1}$. After reaching $400 \mathrm{~K}$ curve exhibits negative slope which indicates the volume contraction. The most probable explanation of such behavior is structural relaxation by annealing-out of the quenched-in free volumes which are introduced when casting the rod sample [8]. Similar results on different metallic glasses were reported in [7]. Negative volume changes last until reaching the glass transition temperature $T_{g}$ at which an abrupt increase of the slope is observed. The volume expansion between $T_{g}$ and $T_{x}$ is linear and yields the slope of $\alpha_{\mathrm{th}}=14.6 \pm 0.3 \times 10^{-5} \mathrm{~K}^{-1}$, which is almost four times larger as compared with the slope obtained below $400 \mathrm{~K}$. After reaching $T_{x}$ expansion curve reveals discontinuity which has no physical meaning and indicates that the position of the principal diffuse peak has dramatically changed due to superposition with the Bragg peaks. Further one can observe that the values for $T_{g}$ and $T_{X}$ obtained from DSC and in situ XRD are in perfect agreement. Fig. 2b shows the thermal expansion curve obtained by tracing the peak position of the principal diffuse peak when heating and cooling in the molten state, above the melting temperature $T_{m}$. One may conclude that the linear behavior of the volume expansion 


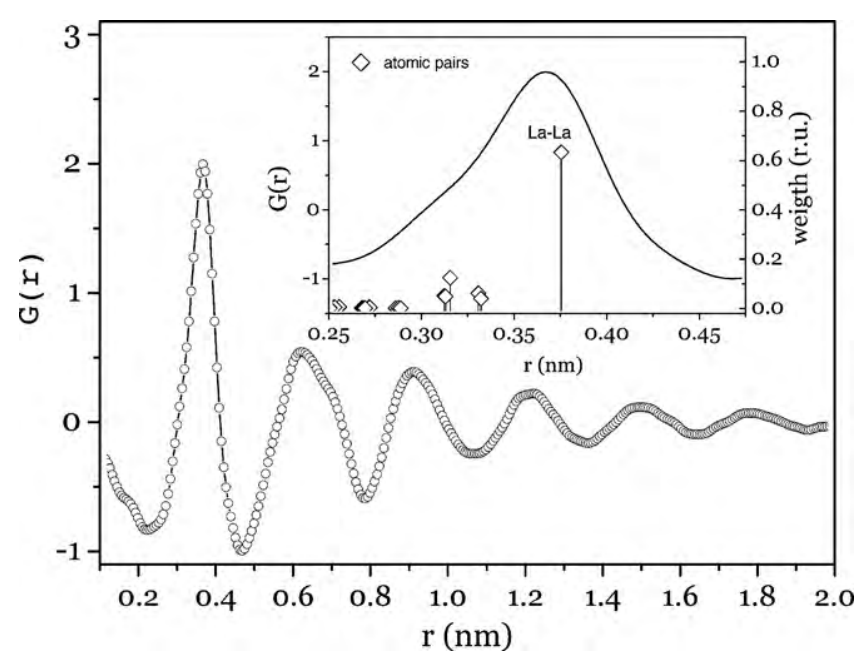

Fig. 3. Reduced pair distribution function $G(r)$ for as-casted La-based rod determined at room temperature. The inset shows detailed view of the first coordination shell. Atomic pair distances were calculated as a sum of nominal atomic radii.

reveals no traces of thermal hysteresis and suggests that heating and cooling of La-based BMG above liquidus temperature $T_{L}$ is fully reversible process. Obtained coefficient of the volume thermal expansion $\alpha_{\mathrm{th}}=9.6 \pm 0.1 \times 10^{-5} \mathrm{~K}^{-1}$ is one and half times smaller than the value obtained within the supercooled liquid region $\Delta T_{x}$.

So far we discussed results based on analysis in reciprocal space, utilizing only part of a data in the vicinity of the principal diffuse peak. In order to get the local atomic structure of the as-casted sample and observe how it is affected upon applying thermal load we conducted real space analysis by observing the changes on reduced pair distribution function $G(r)$ defined by Eq. (2). Fig. 3 shows reduced pair distribution function $G(r)$ of as-casted Labased rod at the room temperature, prior heating experiment. For $\mathrm{La}_{62} \mathrm{Al}_{14}\left(\mathrm{Cu}_{5 / 6} \mathrm{Ag}_{1 / 6}\right)_{14} \mathrm{Ni}_{5} \mathrm{Co}_{5} \mathrm{BMG}$, there are 21 possible nearestneighbor partials with corresponding X-ray weights. However from the type and concentration of the constituent elements it is clear that La-La pairs represent dominant atomic pairs which determine the shape of the first coordination shell. As can be seen from the inset in Fig. 3, La-La atomic pairs form the maximum of $G(r)$ at $r=0.37 \mathrm{~nm}$, whereas the left shoulder is formed by less pronounced atomic pairs of type $\mathrm{La}-\mathrm{M}(\mathrm{M}=\mathrm{Al}, \mathrm{Cu}, \mathrm{Ag}, \mathrm{Ni}, \mathrm{Co})$ with almost no contribution from other remaining atomic pairs. To trace the changes in the local atomic structure upon heating one could convert all collected XRD patterns into corresponding structure factors $S(q)$ using Eq. (1) and subsequently perform its Fourier transform using Eq. (2). Though such approach is possible, it is quite demanding to properly apply set of different corrections on a large number of diffraction patterns in order to get reliable data. This is especially true when correcting for incoherently scattered intensity due to Compton effect and multiple scattering. One way to overcome such difficulty is to perform difference analysis [9]. Assuming that the sample cross-section and the flux on the sample have not changed (after applying normalization due to the beam decay both assumptions are full filled in the present study), the difference in coherently scattered intensity between two consecutive scans can be simplified to $\Delta I^{\mathrm{coh}}(q) \simeq C \Delta I^{\text {obs }}(q)$ where $C$ is the normalization constant which should be used to scale observed intensity to the estimated contributions from the coherent and incoherent scattering. When combined with the Eq. (1) the change in $S(q)$ can be written as $\Delta S(q)=C \Delta I^{\mathrm{obs}}(q) /\langle f\rangle^{2}$. This equation directly links the changes in observed intensities with the changes in $S(q)$ and thus helps to bypass corrections such as sample absorption, experiment

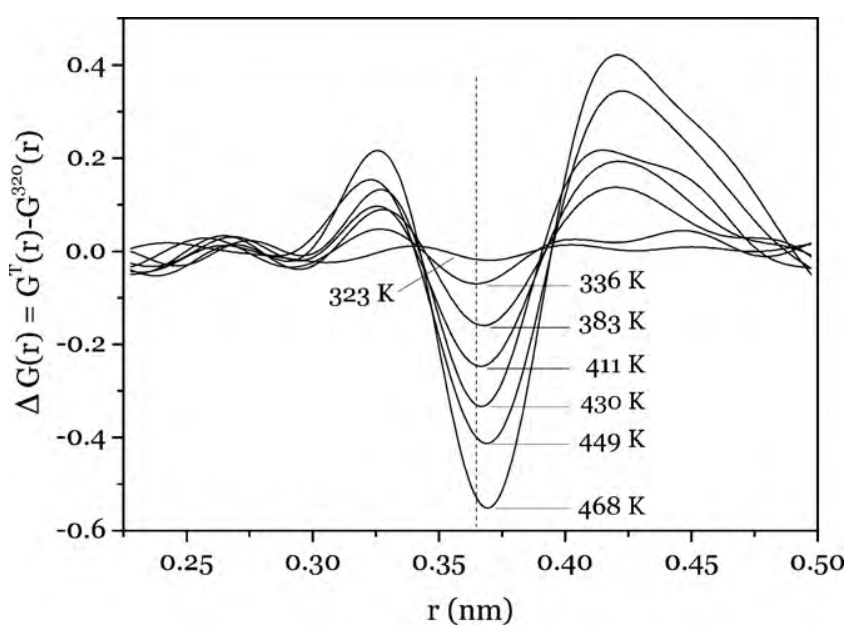

Fig. 4. Temperature behavior of the differenced data sets for $\Delta G(r)$ calculated according Eq. (4). For better clarity only few curves are included. Vertical dashed line depicts the position $\left(r=0.37 \mathrm{~nm}^{-1} \mathrm{~nm}^{-1}\right)$ of the peak maximum in $G(r)$ determined at room temperature.

geometry, beam polarization and incoherent scattering (Compton and multiple scattering), which are usually applied when calculating the total structure factor. The change in the reduced pair distribution function is then given by

$\Delta G(r)=\frac{2}{\pi} \int_{0}^{\infty} q[\Delta S(q)] \sin (q r) \mathrm{d} q$.

Fig. 4 shows the temperature evolution of $\Delta G(r)=G^{T}(r)-$ $G^{320}(r)$ between $320 \mathrm{~K}$ and the onset of crystallization $T_{X}=482 \mathrm{~K}$. One can see that the peak corresponding to the first coordination shell ( $r$-range $0.25-0.5 \mathrm{~nm}$ ) shifts toward higher $r$-values and progressively decreases its amplitude with increasing temperature as manifested by shift and intensity lowering of the difference curve $\Delta G(r)$ between 0.34 and $0.39 \mathrm{~nm}$. Furthermore appearance of the two positive side wings indicates simultaneous broadening of the first coordination peak which also means an increase of the number of atoms having separations $0.3-0.34 \mathrm{~nm}$ and $0.39-0.49 \mathrm{~nm}$, respectively. Significantly larger area of the right wing on $\Delta G(r)$ indicates that atoms prefer to have larger separations with increasing the temperature.

\section{Conclusions}

In situ X-ray diffraction experiments on La-based BMG provided data about its thermal stability. Obtained results are in a good agreement with DSC measurements. Tracing the position of the principal diffuse peak we obtained the thermal expansion curve. About $20 \mathrm{~K}$ below $T_{g}=422 \mathrm{~K}$ expansion curve exhibits strong deviation from linearity which suggests contraction of the mean atomic volumes. Such behavior can be explained by annealing-out of the quenched-in free volumes which are introduced when casting the rod sample. Reaching glass transition temperature $T_{g}$ is accompanied by an abrupt change of the volume thermal expansion coefficient $\alpha_{\mathrm{th}}$. Real space analysis reveals the change of the first coordination shell suggesting redistribution of atoms in the nearest neighborhood.

\section{Acknowledgement}

This research was supported by the Zhejiang UniversityHelmholtz cooperation fund. 


\section{References}

[1] A. Inoue, Acta Mater. 48 (2000) 279.

[2] A. Inoue, T. Zhang, Mater. Trans. JIM 37 (1996) 185.

[3] Q.K. Jiang, G.Q. Zhang, L.Y. Chen, J.Z. Wu, H.G. Zhang, J.Z. Jiang, J. Alloy Compd, $424(2006) 183$.

[4] A.P. Hammersley, S.O. Svensson, M. Hanfland, A.N. Fitch, D. Häusermann, High Press. Res. 14 (1996) 235.
[5] T. Egami, S.J.L. Billinge, Underneath the Bragg Peaks: Structural analysis of Complex Materials, Pergamon Press, Elsevier, Oxford, England, 2003.

[6] T.E. Faber, J.M. Ziman, Philos. Mag. 11 (1965) 153.

[7] A.R. Yavari, A. Le Moulec, A. Inoue, N. Nishiyama, N. Lupu, E. Matsubara, W.J. Botta, G. Vaughan, M. di Michiel, Å. Kvick, Acta Mater. 53 (2005) 1611.

[8] A.I. Taub, F. Spaepen, Acta Metall. 28 (1980) 1781.

[9] M.J. Kramer, J. Appl. Cryst. 40 (2007) 77. 